\title{
SEGURANÇA DO PACIENTE: AVALIAÇÃO DO SISTEMA DE MEDICAÇÃO POR ENFERMEIROS UTILIZANDO ANÁLISE FOTOGRÁFICA
}

Camila Dannyelle Fernandes Dutra Pereiraํ․ Francis Solange Vieira Tourinho ${ }^{2}$, Viviane Euzébia Pereira Santos ${ }^{3}$

Objetivo: identificar como enfermeiros avaliam a Segurança do Paciente no sistema de medicação através da análise fotográfica. Metodologia: estudo descritivo e quantitativo desenvolvido em um hospital de ensino, com amostra de 34 enfermeiros das unidades de internação. A coleta dos dados utilizou o método fotográfico. Abordou-se a avaliação da segurança em 10 fotografias através da escolha de um número entre 1 (muito inseguro) e 10 (muito seguro) e a análise dos dados foi realizado no Statistical Package for the Social Scienses versão 20.0. Resultados: adaptando os escores atribuidos à escala, foi possivel identificar entre as fotografias: três Totalmente Inseguras (Grau 1), três Inseguras (Grau 2), três Seguras Parcialmente (Grau 3) e uma Segura (Grau 4). Conclusão: o estudo evidencia fragilidades no sistema de medicação, o que exige que a Enfermagem revise seu processo de trabalho para prevenir os erros, fundamentando o cuidado na Segurança do Paciente.

Descritores: Enfermagem, Segurança do Paciente, Sistemas de Medicação.

\section{PATIENT SAFETY: NURSES' EVALUATION OF THE MEDICATION SYSTEM THROUGH PHOTOGRAPHIC ANALYSIS}

Objective: this study aimed to identify how nurses assess Patient Safety in the medication system through photographic analysis. Methodology: a descriptive and quantitative study conducted in a teaching hospital, with a sample of 34 nurses from inpatient units. Data collection used the photographic method. This study addressed the safety assessment in 10 photographs by choosing a number in a scale from 1 (very unsafe) to 10 (very safe) and data analysis was performed using the Statistical Package for Social Scienses version 20.0. Results: by adapting the scores attributed to the scale, we were able to identify among the photographs: three Totally Unsafe (Level 1), three Unsafe (Level 2), three Partially Safe (Level 3), and one Safe (Level 4). Conclusion: the study highlights the weaknesses in the medication system, which requires that nursing review its work process to prevent errors, basing the care on Patient Safety.

Descriptors: Nursing, Patient Safety, Medication Systems.

\section{SEGURIDAD DEL PACIENTE: EVALUACIÓN DE LO SISTEMA DE MEDICAMENTOS PARA ENFERMEROS UTILIZANDO ANÁLISIS DE IMAGEN}

Objetivo: tuvo como objetivo identificar cómo los enfermeros evalúan la seguridad del paciente en el sistema de medicamentos a través de análisis fotográfico. Metodología: estudio descriptivo y cuantitativo realizado en un hospital universitário, con una muestra de 34 enfermeros de las unidades de hospitalización. La recolección de datos utiliza el método fotográfico. Este estudio abordó la evaluación de la seguridad en 10 fotografias por la elección de un número entre 1 (muy inseguro) a 10 (muy seguro) y análisis de datos se realizó mediante el Statistical Package for Social Scienses version 20.0. Resultados: adaptación de las puntuaciones atribuidas a la escala pudo identificar entre las fotografias: tres Totalmente Inseguro (Grado 1) tres Inseguro (Grado 2), tres Parcialmente Seguro (Grado 3) y una Segura (Grado 4). Conclusión: el estudio pone de relieve las debilidades en el sistema de medicamentos, lo que requiere que la enfermería revisar su proceso de trabajo para evitar errores, basando la atención sobre la Seguridad del Paciente.

Descriptores: Enfermería, Seguridad del Paciente, Sistemas de Medicación.

${ }^{1}$ Enfermeira. Mestre em Enfermagem pelo Programa de Pós-Graduação em Enfermagem da Universidade Federal do Rio Grande do Norte (UFRN). E-mail: camilafernandes_enf@hotmail.com

${ }^{2}$ Enfermeira. Doutora. Professora Adjunto da Universidade Federal de Santa Catarina (UFSC). Programa de Pós-Graduação em Enfermagem, UFSC. ${ }^{3}$ Enfermeira. Doutora. Professora Adjunto da UFRN, Programa de Pós-Graduação em Enfermagem, UFRN. 


\section{INTRODUÇÃO}

A Segurança do Paciente vem sendo tema de destaque na área da saúde, o que culmina com o desenvolvimento de estudos e, consequentemente, de evidências científicas ${ }^{(1)}$. Os erros de medicação podem determinar inúmeras consequências: para os pacientes, agravos à saúde e alterações clínicas; para o sistema, aumento do tempo de internação e elevação dos custos com procedimentos ${ }^{(2.3)}$.

No cenário da Enfermagem, a preocupação com a Segurança do Paciente está presente e pode ser evidenciada pelo movimento social que em 2008 culminou com a Rede Brasileira de Enfermagem e Segurança do Paciente (REBRAENSP), objetivando cuidados seguros ${ }^{(1)}$. Em 2013, o Ministério da Saúde (MS) juntamente à Agência Nacional de Vigilância Sanitária (ANVISA), criaram, através da Portaria no 529 do MS, de 10 de abril de 2013, o Programa Nacional de Segurança do Paciente (PNSP)(4).

Nesse contexto de segurança, o sistema de medicação merece atenção, já que os erros relacionados aos medicamentos representam um desafio de saúde pública, afetando principalmente, os pacientes em ambientes hospitalares ${ }^{(5)}$.

Os erros relacionados aos medicamentos são classificados como eventos adversos evitáveis, estando frequentemente presentes na realidade hospitalar; em média, em um paciente hospitalizado ocorre, pelo menos, um erro de medicação/ dia(b). A Harvard Medical Pratice realizou uma pesquisa em instituições hospitalares relacionada aos eventos adversos e identificou que os erros de medicação ocorrem em uma proporção de um para cada cinco eventos, sendo assim, os que mais acometem os pacientes nos serviços de saúde $e^{(7)}$

Diante disso, percebe-se que este sistema é complexo, composto por processos que demandam uma equipe multidisciplinar, envolvendo atividades de diversos profissionais( ${ }^{(8)}$

Nessa equipe de saúde, destaca-se a Enfermagem como sujeito ativo nas instituições hospitalares e como último elo da cadeia, podendo, assim, atuar de forma a evitar os erros ${ }^{(9)}$.

Considerando que a enfermagem está envolvida diretamenteno sistema de medicação, este estudo justifica-se já que a avaliação da Segurança do Paciente nas etapas deste sistema por enfermeiros auxilia a identificação de possiveis riscos. Além disso, contribui, consequentemente, para que os profissionais conheçam e realizem uma reflexão crítica de suas práticas diárias relacionadas aos medicamentos e desenvolvam uma assistência de qualidade, competente e criativa, baseada nos princípios de segurança. Frente à importância dessa temática, este estudo objetivou identificar como enfermeiros avaliam a Segurança do Paciente no sistema de medicação através da análise fotográfica.

\section{METODOLOGIA}

Estudo descritivo com abordagem quantitativa, desenvolvido em um hospital de ensino no Rio Grande do Norte/RN, Brasil. A população foi composta pelos 42 enfermeiros das unidades de internação do referido hospital. Todos os sujeitos foram arrolados, no entanto, constituiu uma amostra do tipo não probabilística intencional, resultando em 34 profissionais. Os critérios de elegibilidade utilizados foram ser enfermeiro servidor público no referido hospital e aceitar participar livremente da pesquisa.

As determinações da Resolução no 196/1996 do Conselho Nacional de Saúde (CNS), revisadas pela Resolução no 466/2012 (CNS), foram legalmente cumpridas, respeitando os aspectos éticos das pesquisas com seres humanos, sob o parecer do Comitê de ética (0098.0.051.294-11)(10).

Para coleta dos dados, utilizou-se o método fotográfico através da Técnica de Análise Fotográfica adaptada de Patrícia Marck. Consiste no uso da fotografia digital proveniente do desenvolvimento do Digital Photography Scoring Tool (DPST) do Programa de Pesquisa e Restauração da Faculdade de Enfermagem da Universidade de Alberta, Canadá, em parceria com outros investigadores do Programa de Pesquisa e Restauração em Sistemas Modernos de Saúde ${ }^{(11.12)}$. Esse processo de coleta desenvolveu-se em duas fases: 1- captura das fotografias de forma aleatória, resultando em 282 imagens e posteriormente a seleção e tratamento das fotografias digitais, as quais foram reduzidas a 10 imagens aleatoriamente utilizando o programa Microsoft Excel 2010. Essas fotografias receberam tratamento para preservar o anonimato dos profissionais e pacientes fotografados e foram reveladas, organizadas e enumeradas em pasta fichário; 2- enfermeiros responderam o questionário estruturado de coleta dos dados, dividido em perfil sócio profissional e DPST.

O DPST foi composto por: a) descrição da avaliação geral da área fotografada em relação à segurança do paciente; b) avaliação da segurança da cena apresentada na fotografia através da escolha de um número entre 1 (muito inseguro) e 10 (muito seguro).

O tratamento dos dados foi realizado no Statistical Package for the Social Scienses (SPSS) versão 20.0, utilizando medida de tendência central (média aritmética) e de dispersão (desvio padrão), como também valor de mínimo e de máximo. $\mathrm{Na}$ apresentação dos dados, foi utilizada a escala Likert. Assim, os escores de 1 a 10 foram representados através de cinco graus de variação, que compuseram a escala: Grau 1 (pontuação 1 e 2) - Inseguro Totalmente (IT); Grau 2 (pontuação 3 e 4) - Inseguro (I); Grau 3 (pontuação 5 e 6) Seguro Parcialmente (SP); Grau 4 (pontuação 7 e 8) - Seguro (S); e Grau 5 (pontuação 9 e 10) - Seguro Totalmente (ST). 


\section{RESULTADOS}

No que concerne ao perfil sócio profissional, os enfermeiros do estudo foram predominantemente do sexo feminino, correspondendo a 31 profissionais (91,2\%). A idade destes participantes variou entre o mínimo de 23 anos e o valor máximo de 65 , sendo estas categorizadas em quatro faixas etárias: 23-33 (8 - 23,5\%); 34-43 (14 - 41\% ); 44-53 (5 $15 \%$ ); 54-65 (7 - 20,5\%)

Dezenove (55,9\%) enfermeiros eram especialistas, sendo oito em assistência, oito em gestão e três em educação; 10 (29,4\%) possuíam mestrado, sendo nove na área da enfermagem e um na de saúde coletiva; 2 (5,9\%) fizeram residência em enfermagem e $3(8,8 \%)$ possuíam apenas a graduação em enfermagem. Referindo-seaotempo de serviço, este variou de menos de um ano até 36 anos, com destaque ao período de 10 a 18 anos de serviço, correspondendo a 11 $(32,3 \%)$ profissionais.

Vinte e nove (85,3\%) enfermeiros afirmaram atuar exclusivamente no referido hospital. Acerca do conhecimento destes profissionais sobre o tema Segurança do Paciente, quase todos responderam que conheciam a temática, o que caracterizou um total de 31 (91,2\%) enfermeiros. Assim, 3 $(8,8 \%)$ participantes disseram que não conheciam sobre o assunto. Para a compreensão da avaliação da segurança das fotografias, este estudo considerou a descrição das imagens apresentadas no Quadro $\mathrm{Ol}$.

No que se refere aos valores de mínimo e de máximo, as imagens obtiveram variações nas suas avaliações, podendo destacar que a de menor variação de escores foi a fotografia 5 , avaliada pelos enfermeiros com os escores entre o mínimo de 1 e máximo de 6

Visualizou-se que a fotografia 9 obteve a maior média aritmética $(7,00)$, sendo a imagem considerada mais segura na avaliação dos enfermeiros. A fotografia 6 foi a de menor média (3,12), o que significou que os profissionais, em sua maioria, a consideraram como a mais insegura. Isto pode ser comprovado levando em consideração a moda dos escores destas imagens, nas quais os valores que mais se repetiram foi 7 e 1 , respectivamente.

A fotografia 1 foi classificada como Segura Parcialmente de acordo com 11 (32,3\%) enfermeiros que atribuíram o escore 5 ou 6 na sua avaliação. A segunda imagem foi considerada como Insegura por 11 (32,3\%) profissionais que a avaliaram com os valores de 3 ou 4. Referindo-se às imagens 3 e 4, estas foram classificadas como Seguras Parcialmente por 11 (32,3\%) e 12 (35,3\%) participantes, respectivamente. A quinta fotografia foi considerada Insegura por 14 (41,2\%) enfermeiros que avaliaram a cena entres os escore de 3 ou 4. As imagens 6 e 7, respectivamente, foram classificadas como Inseguras Totalmente por $16(47 \%)$ e 15 (44,1\%) profissionais. A fotografia 8 foi considerada como Insegura segundo 12 (35,3\%) enfermeiros. A nona imagem foi a única classificada como Segura de acordo com a avaliação de 14 (41,2\%) participantes que a avaliaram utilizando o escore de 7 ou 8. E por fim, a imagem 10 foi considerada como Insegura Totalmente segundo 18 (53,0\%) profissionais.

Quadro - Descrição das fotografias utilizadas no estudo. Natal/RN, 2013.

\begin{tabular}{|ll|}
\hline Fotografias & Descrição \\
\hline Fotografia l & $\begin{array}{l}\text { Dispensação dos medicamentos prescritos, em } \\
\text { doses unitárias. Entrega realizada pela farmácia e } \\
\text { recebida pela enfermagem. }\end{array}$ \\
\hline
\end{tabular}

Fotografia 2 Dispensação dos medicamentos prescritos, em doses unitárias. Entrega realizada pela farmácia e recebida pela enfermagem.

Fotografia 3 Prescrição médica digitalizada referente a um paciente internado com acréscimos manuscritos pelos médicos

Fotografia 4 Carro com gavetas de plástico, móveis e enumeradas para armazenamento dos medicamentos de acordo com os leitos; gavetas maiores para soros fisiológicos.

Fotografia 5 Gaveta de madeira com divisórias para o armazenamento de medicamentos psicotrópicos em excesso, sem chaves e mistura de medicamentos nas divisórias.

Fotografia 6 Separação e preparo prévio dos medicamentos. Administração via oral em copos de plástico com etiquetas adesivas para identificação; Via endovenosa, a ampola e a água bidestilada agrupadas e identificadas com etiqueta adesiva.

Fotografia 7 Separação prévia do medicamento para preparo/administração. Identificação com etiqueta adesiva.

Fotografia 8 Administração de medicamento endovenoso em acesso venoso periférico em membro superior direito pela técnica de enfermagem sem uso de equipamento de proteção individual (EPI) Paciente na cadeira.

Fotografia 9 Administração de medicamento endovenoso em acesso venoso periférico em membro superior esquerdo pela técnica de enfermagem com uso de EPI. Paciente no leito.

Fotografia 10 Descarte de seringas na caixa de perfurocortante, mal fechada e com seringas saindo pela entrada do recipiente.

Fonte: Dados próprios do estudo.

\section{DISCUSSÃO}

$\mathrm{Na}$ prática da Enfermagem, é imprescindivel que o atendimento desenvolvido seja de qualidade e seguro. Isso 
porque, além de ser um direito legalmente estabelecido, os pacientes quando submetidos ao ambiente hospitalar, esperam ter seus problemas resolvidos mediante cuidados bem realizados ${ }^{(13)}$

A Enfermagem atua diretamente nas etapas finais do sistema de medicação: preparo e administração dos medicamentos prescritos. Neste estudo, a fotografia 6 (Figura 01) retratou a etapa de separação e preparo, sendo considerada pelos enfermeiros como a mais insegura, já que obteve a menor média aritmética, o que condiz com a classificação Insegura Totalmente.

Mesmo sendo um procedimento complexo, a Enfermagem, muitas vezes, realiza o preparo dos medicamentos como uma atividade simples, dificultando a prevenção dos erros e a segurança na terapia medicamentosa ${ }^{(9)}$. Os fármacos devem ser preparados no momento correto, pois realizar a atividade antecipadamente pode gerar muitos riscos, como troca de medicamentos e alteração do efeito terapêutico ${ }^{(13)}$

Figura 01 - Fotografia 6: imagem mais insegura na avaliação dos enfermeiros. Natal/RN, 2013.

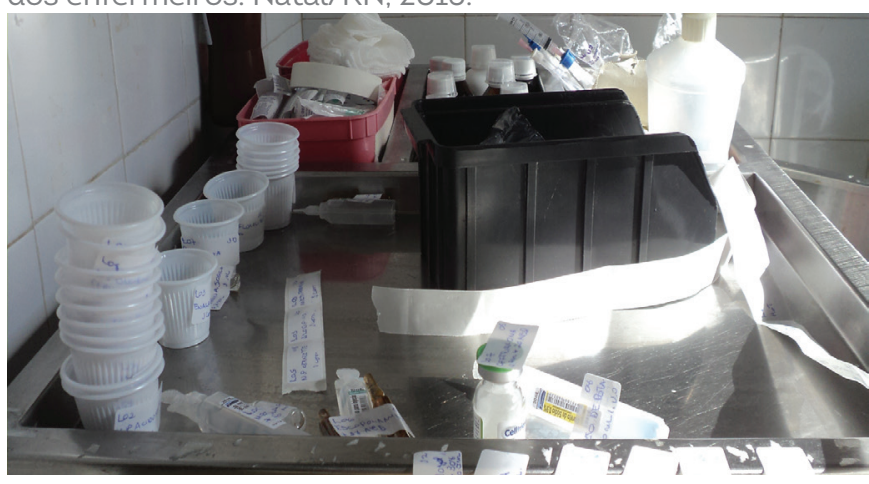

A fotografia 9 (Figura 02) evidenciou a realização desse procedimento e, neste estudo, foi a imagem que obteve a maior média aritmética, demonstrando que foi avaliada pelos profissionais como a mais segura, o que corrobora com a Escala de Likert, sendo a única imagem classificada como Segura (S)

Figura 02 - Fotografia 9: imagem mais segura na avaliação dos enfermeiros. Natal/RN, 2013.

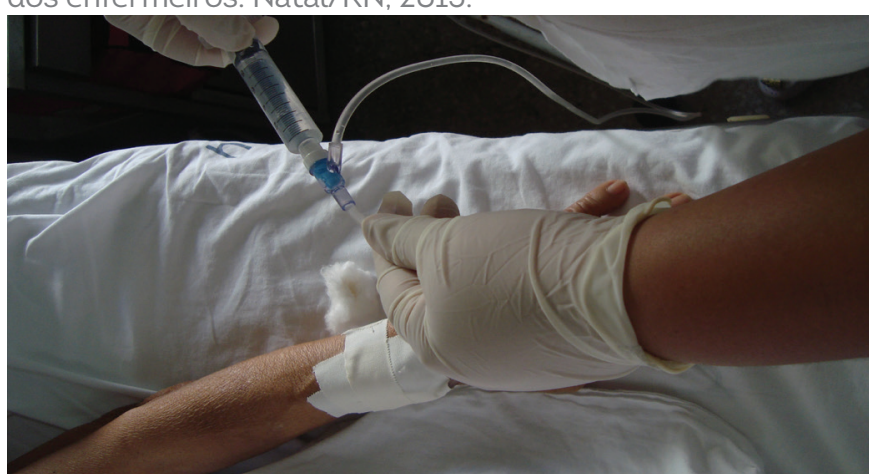

No Brasil, a administração de medicamentos é legalmente uma atribuição da Enfermagem e, dentre as responsabilidades dessa equipe, é considerada como uma das mais importantes, o que exige que esse procedimento seja desenvolvido de modo seguro, a fim de prevenir a ocorrência de erros ${ }^{(14,15)}$

Muitas vezes, percebe-se o despreparo dos profissionais e a falta de atenção à técnica e às condutas de segurança ${ }^{(14)}$. Um instrumento utilizado internacionalmente na Enfermagem para diminuir os erros é a regra dos "Nove Certos". É importante ressaltar que esta regra não garante que os erros durante a administração dos medicamentos não vão ocorrer, mas auxilia para o desenvolvimento de um cuidado adequado baseado em uma assistência efetiva e segura(13-16).

Ainda para garantia da segurança, um aspecto de destaque na Figura 02 é o uso de Equipamento de Proteção Individual (EPI) pelo profissional de Enfermagem que administra o medicamento. O uso do EPI, nesse caso a luva, além de proteger o paciente, serve como aliado dos profissionais envolvidos nos procedimentos, funcionando como barreira para a prevenção de acidentes. Para isso, é importante uma educação permanente visando autocuidado, e reflexão sobre a ocorrência de acidentes e erros devido a falta de $\mathrm{EPI}^{(17)}$

Por atuar principalmente nessas etapas finais, a Enfermagem funciona como elo final. Essa situação faz com que muitos dos erros cometidos durante todo o sistema sejam atribuidos aos profissionais de Enfermagem, além de que a responsabilidade aumenta, já que pode ser a última chance de evitar o erro e garantir a segurança na assistência $^{(18)}$

O sistema de medicação é formado por um conjunto de processos que são planejados e realizados por uma equipe multiprofissional que desempenha suas atividades para restabelecimento da saúde do paciente ${ }^{(19)}$. Cabe ao enfermeiro conhecer todo o sistema, desde a prescrição até a administração, para assim, identificar os possiveis riscos e evitar que os erros ocorram, sendo um profissional de grande importância para segurança do paciente ${ }^{(7,20)}$.

\section{CONCLUSÃO}

No que concerne às avaliações dos enfermeiros acerca das fotografias, foi possivel identificar algumas divergências de avaliação entre os participantes. A avaliação resultou em fotografias Totalmente Inseguras, Inseguras, Seguras Parcialmente e Segura, cabendo salientar que não houve uma fotografia considerada pelos profissionais, em sua maioria, como Segura Totalmente.

Apreende-se que o sistema de medicação é complexo, caracterizado pela atuação de diversos profissionais e 
marcado por fragilidades. Nesse panorama, é importante que a Enfermagem revise seu processo de trabalho e desenvolva uma assistência na terapia medicamentosa a fim de promover um cuidado adequado e de qualidade, visando à prevenção de erros e fundamentando-se, assim, nos princípios propostos pela Segurança do Paciente.
REFERÊNCIAS

1. Cassiani SHB. Enfermagem e a Pesquisa sobre Segurança dos Pacientes. Acta Paul Enferm [online]. 2010 [Acesso 2016 Abr 04]; 23(6):7-8. Disponivel em: http://www.scielo.br/pdf/ape/v23n6/01.pdf

2. National Coordinating Council for Medication Error Reporting and Prevention - NCCMERP. Index for categorizing medication errors. 2001. Disponivel em: http://www.nccmerp.org/medErrorCatIndex.html.

3. Anacleto TA, Rosa MB, Neiva HM, Martins MAP. Farmácia Hospitalar: Erros de medicação. Pharmacia Brasileira: 2010.

4. Ministério da Saúde (BR). Coletiva Segurança do Paciente. Brasilia (DF): MS; 2013. [Acesso 2013 Ago 04]: Disponivel em: http://portalsaude. saude.gov.br/portalsaude/arquivos/pdf/2013/Abr/O1/PPT_COLETIVA_ SEGURANCA_PACIENTE_FINAL.pdf.

5. Gimenes FRE, Mota MLS, Teixeira TCA, Silva AEBC, Opitz SP, Cassian SHB. Segurança do paciente na terapêutica medicamentosa e a influência da prescrição médica nos erros de dose. Rev Latino-Am Enfermagem. 2010 Nov-Dez [Acesso 2016 Abr 04]; 18(6):[07 telas]. Disponivel em: http://www. scielo.br/pdf/rlae/v18n6/pt_03.pdf

6. Silva LD. Camerini FG. Análise da administração de medicamentos intravenosos em hospital da rede sentinela. Texto Contexto Enferm. 2012 Jul-Set [Acesso 2016 Abr 04]; 21(3):633-41. Disponivel em: http://www. scielo.br/pdf/tce/v2ln3/v2ln3a19.pdf

7. Lisby M, Nielsen LP, Brock B, Mainz J. How are medication errors dened? A systematic literature review of denitions and characteristics. International Journal for Quality in Health Care. 2010 [Acesso 2016 Abr 04]; 22(6):507518. Disponivel em: http://intahc.oxfordjournals.org/content/22/6/507.full. pdf+html

8. Bohomol E, Ramos LH. Erro de medicação: importância da notificação nogerenciamento da segurança do paciente. Rev Bras Enferm. 2007 JanFev [Acesso 2016 Abr 04]; 60(1):32-6. Disponivel em: http://www.scielo.br/ pdf/reben/v60nl/aO6v60nl.pdf

9. Camerini FG, Silva LD. Segurança do Paciente: análise do preparo de medicação intravenosa em hospital da rede sentinela. Texto Contexto Enferm. 2011 Jan-Mar [Acesso 2013 Dez 03]; 20(1):41-9. Disponivel em: http://www.scielo.br/pdf/tce/v20nl/05.pdf

10. Ministério da Saúde (BR), Conselho Nacional de Saúde. Resolução nํ 466, de 12 de dezembro de 2012. Trata de pesquisas em seres humanos e atualiza a Resolução 196/1996. Brasilia (DF): MS; 2012. Disponivel em: http:// conselho.saude.gov.br/resolucoes/2012/Reso466.pdf

11. Raduenz AC, Hoffmann P, Radunz V, Dal Sasso GTM, Maliska ICA, Marck PB. Cuidados de enfermagem e segurança do paciente: visualizando a organização, acondicionamento e distribuição de medicamentos com método de pesquisa fotográfica. Rev Latino-Am Enfermagem. 2010 NovDez [Acesso 2016 Abr 04]; 18(6):[10 telas]. Disponivel em: http://www.scielo. $\mathrm{br} / \mathrm{pdf} / \mathrm{rlae} / \mathrm{v} 18 \mathrm{n} 6 / \mathrm{pt} \_02 . p d f$

12. Marck P. Keehan L, Morgan Eckley W, Verluy R. The Development of a Digital Photography Scoring Tool. 2005-2006. Disponivel em: http://www. nurs.ualberta.ca/safersystems/projects.htm

13. Elliott M. Liu Y. The nine rights of medication administration: an overview. British Journal Nursing. 2010 [Acesso 2016 Abr 06]; 19(5). Disponivel em: http://rn-transition-programs.wikispaces.com/file/ view/9RightsMedAdm2010.pdf

14. Fakih FT, Freitas GF, Secoli SR. Medicação: aspectos ético-legais no âmbito da enfermagem. Rev Bras Enferm. 2009 [Acesso 2016 Abr 02]; 62(1):132-35. Disponivel em: http://www.scielo.br/pdf/reben/v62nl/20.pdf

15. Franco JN, Ribeiro G, Innocenzo MD, Barros BPA. Percepção da equipe de enfermagem sobre os fatores causais de erros de administração de medicamentos. Rev Bras Enferm. 2010 [Acesso 2016 Abr 04]; 63(6). Disponivel em: http://www.scielo.br/pdf/reben/v63n6/09.pdf

16. Munn Z, Scarborough A, Pearce S, Mc Arthur A, Kavanagh S, Girdler M et al. The implementation of best practice in medication administration across a health network: a multisite evidence-based audit and feedback project. The JBI Database of Systematic Reviews and Implementation Reports. 2016 [Acesso 2016 Abr 06] [S.L.], 13(12): 335. Jan. Disponivel em: <http:// joannabriggslibrary.org/index.php/jbisrir/article/view/2779/2778>.

17. Senna MH, Silva CC, Gelbcke FL, Anders JC, Mesquita MPL. A segurança do trabalhador de enfermagem na administração de quimioterápicos antineoplásicos por via endovenosa. Revista Enfermagem UERJ, 2005 [Acesso 2016 Abr 06] 22(5), 649-55. Disponivel em: http://www.facenf.uerj. br/v22n5/v22n5all.pdf

18. Fassarella CS, Bueno AAB, Souza ECC. Segurança do paciente no ambiente hospitalar: os avanços na prevenção de eventos adversos no sistema de medicação. Rev Rede Cuidados Saúde. 2013 [Acesso 2015 Dez 05]; 7(1). Disponivel em: http://publicacoes.unigranrio.edu.br/index.php/ rcs/article/viewFile/1897/907Florianópolis: Universidade Federal de Santa Catarina. Programa de Pós Graduação em Enfermagem; 2006.

19. Santi T, Beck CLC, da Silva RM, Zeitoune RG. Tonel JZ, do Reis DAM. Error de medicación en un hospital universitario: percepción y factores relacionados. Enferm. Glob. 2014 Jul [Acesso 2016 Abr 06]; 13(35): 160-71. Disponivel em: http://scielo.isciii.es/scielo.php?script=sci_ arttext\&pid=S1695-61412014000300010\&lng=es.

20. Kim MY, Kim YM, Kang SW. A survey and multilevel analysis of nursing unit tenure diversity and medication errors. Journal of Nursing Management. 2016. Disponivel em: http://onlinelibrary.wiley.com/doi/10.1111/jonm.12366/pdf. 